\title{
Elimination of the Cortico-Subthalamic Hyperdirect Pathway Induces Motor Hyperactivity in Mice
}

\author{
Daisuke Koketsu, ${ }^{1}{ }^{\circledR}$ Satomi Chiken, ${ }^{1,2}{ }^{\circledR}$ Tatsuhiro Hisatsune, ${ }^{3}$ Shigehiro Miyachi, ${ }^{4}$ and ${ }^{\circledR}$ Atsushi Nambu ${ }^{1,2}$ \\ ${ }^{1}$ Division of System Neurophysiology, National Institute for Physiological Sciences, Okazaki 444-8585, Japan, ${ }^{2}$ Department of Physiological Sciences, \\ SOKENDAI (The Graduate University for Advanced Studies), Okazaki 444-8585, Japan, ${ }^{3}$ Department of Integrated Biosciences, Graduate School of \\ Frontier Sciences, University of Tokyo, Kashiwa 277-8562, Japan, and ${ }^{4}$ Cognitive Neuroscience Section, Primate Research Institute, Kyoto \\ University, Inuyama 484-8506, Japan
}

The substantia nigra pars reticulata $(\mathrm{SNr})$ is the output station of the basal ganglia and receives cortical inputs by way of the following three basal ganglia pathways: the cortico-subthalamo (STN)-SNr hyperdirect, the cortico-striato-SNr direct, and the cortico-striato-external pallido-STN-SNr indirect pathways. Compared with the classical direct and indirect pathways via the striatum, the functions of the hyperdirect pathway remain to be fully elucidated. Here we used a photodynamic technique to selectively eliminate the cortico-STN projection in male mice and observed neuronal activity and motor behaviors in awake conditions. After cortico-STN elimination, cortically evoked early excitation in the $\mathrm{SNr}$ was diminished, while the cortically evoked inhibition and late excitation, which are delivered through the direct and indirect pathways, respectively, were unchanged. In addition, locomotor activity was significantly increased after bilateral cortico-STN elimination, and apomorphine-induced ipsilateral rotations were observed after unilateral cortico-STN elimination, suggesting that cortical activity was increased. These results are compatible with the notion that the cortico-STN-SNr hyperdirect pathway quickly conveys cortical excitation to the output station of the basal ganglia, resets or suppresses the cortical activity related to ongoing movements, and prepares for the forthcoming movement.

Key words: basal ganglia; hyperdirect pathway; mouse; photodynamic technique; subthalamic nucleus

Significance Statement

The basal ganglia play a pivotal role in the control of voluntary movements, and their malfunctions lead to movement disorders, such as Parkinson's disease and dystonia. Understanding their functions is important to find better treatments for such diseases. Here we used a photodynamic technique to selectively eliminate the projection from the motor cortex to the subthalamic nucleus, the input station of the basal ganglia, and found greatly reduced early excitatory signals from the cortex to the output station of the basal ganglia and motor hyperactivity. These results suggest that the neuronal signals through the cortico-subthalamic hyperdirect pathway reset or suppress ongoing movements and that blockade of this pathway may be beneficial for Parkinson's disease, which is characterized by oversuppression of movements.

Received May 28, 2020; revised May 1, 2021; accepted May 3, 2021.

Author contributions: D.K., S.C., T.H., S.M., and A.N. designed research; D.K. and S.C. performed research; D.K. analyzed data; D.K., S.C., and A.N. wrote the paper.

This work was supported by Grants-in-Aid for Scientific Research "Non-linear Neuro-oscillology" $15 \mathrm{H} 05873$ (to A.N.) from the Ministry of Education, Culture, Sports, Science and Technology, Japan; Grants-in-Aid for Scientific Research 25430021 (to S.C.), and 26250009 and 19 KK0193 (to A.N.) from the Japan Society for the Promotion of Science; Core Research for Evolutional Science and Technology Grant (to A.N.) from the Japan Science and Technology Agency; and the Takeda Science Foundation (to A.N.). We thank K. Miyamoto, S. Sato, K. Awamura, H. Isogai, M. Goto, N. Suzuki, and T. Sugiyama for technical assistance; and Y. Yamagata for critical reading of the manuscript.

The authors declare no competing financial interests.

Correspondence should be addressed to Atsushi Nambu at nambu@nips.ac.jp.

https://doi.org/10.1523/JNEUROSCI.1330-20.2021

Copyright $\odot 2021$ the authors

\section{Introduction}

The basal ganglia are crucial for motor behaviors, such as action selection and motor learning (Graybiel, 2005; Redgrave et al., 2010). The striatum and subthalamic nucleus (STN) are the input stations of the basal ganglia and receive cortical inputs (Fig. 1A). Two pathways that originate from the striatum exert opposing effects on the substantia nigra pars reticulata $(\mathrm{SNr})$ and the internal segment of the globus pallidus [GPi (or the entopeduncular nucleus in rodents)], the GABAergic basal ganglia output stations (Alexander and Crutcher, 1990). The striato-SNr/ GPi direct pathway inhibits SNr/GPi activity, and thereby disinhibits the thalamocortical and brainstem circuitries and releases movements; while the striato-external pallido (GPe)-STN-SNr/ GPi indirect pathway enhances $\mathrm{SNr} / \mathrm{GPi}$ activity, and thereby suppresses the thalamocortical and brainstem circuitries and 
A

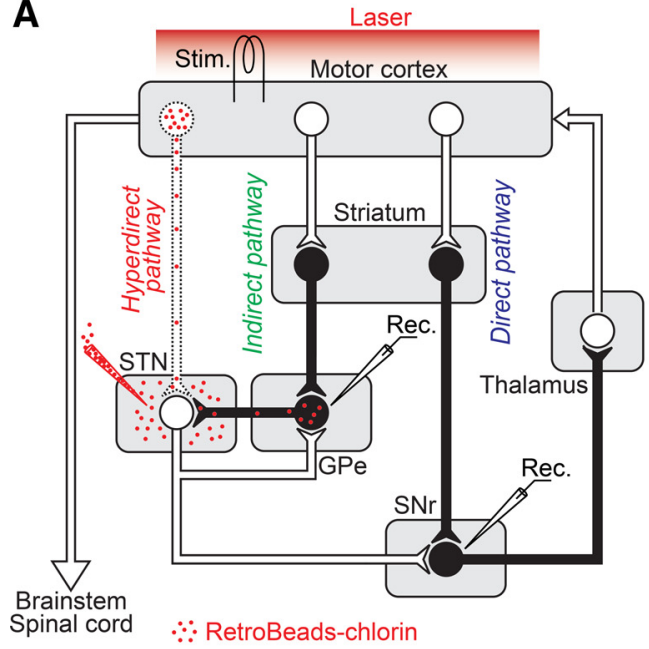

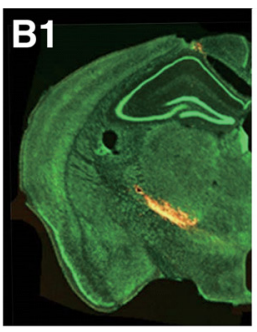

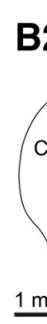

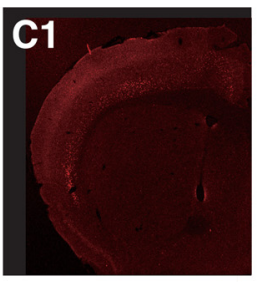

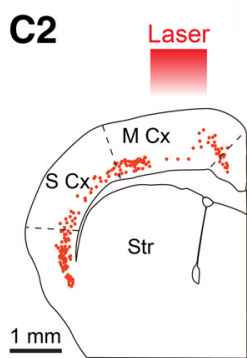

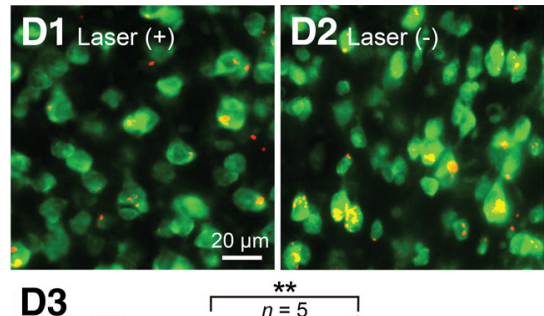

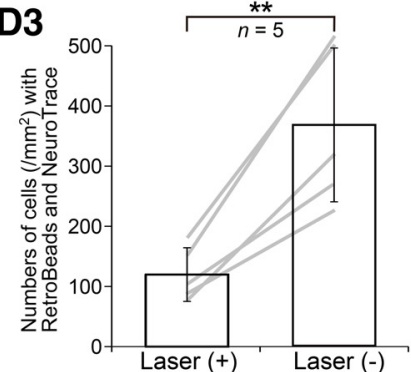

Figure 1. Selective elimination of the cortico-STN projection by a PDT. $A$, Schematic illustration showing the cortico-basal ganglia circuitry composed of the cortico-STN- SNr hyperdirect, cortico-striato-SNr direct, and cortico-striato-GPe-STN-SNr indirect pathways. RetroBeads-chlorin injection, stimulation (Stim.), and recording (Rec.) sites are also indicated. B1, B2, Coronal section containing the STN. B1, Photomicrograph showing that rhodamine-labeled RetroBeads-chlorin (yellow-red) were successfully injected into the STN. Neurons were counterstained with NeuroTrace (green). Similar results were obtained in the other four mice for histologic examinations. B2, Illustration of $\boldsymbol{B} \mathbf{1}$ indicating the injection site with a red color. C1, C2, Coronal section containing the motor cortex 1 month after laser irradiation. C1, Photomicrograph showing that rhodamine-labeled cortico-STN neurons (red) were selectively eliminated in the laser-irradiated cortex, while they were spared from elimination in the surrounding nonirradiated cortex. C2, Illustration of C1 indicating rhodamine-labeled projection neurons with red dots. D1, D2, D3, Double-labeled cells in the motor cortex. D1, D2, Photomicrographs of layer 5 in the laser-irradiated motor cortex (D1) and in the surrounding nonirradiated cortex (D2). Cortico-STN neurons were labeled with rhodamine-RetroBeads (red), while neurons were stained with NeuroTrace (green). D3, The numbers of double-labeled cells were counted in the laser-irradiated cortex $[$ Laser $(+)]$ and in the surrounding nonirradiated cortex [Laser $(-)]$ in five mice. Data are expressed as the mean \pm SD. Gray lines show the data from individual mice. ${ }^{* *} p=0.007$ significantly different from one another, two-tailed paired $t$ test. Cx, Cortex; $M$ Cx, motor cortex; $S$ Cx, somatosensory cortex; Str, striatum; Th, thalamus.

movements (Kravitz et al., 2010; Sano et al., 2013; Chiken et al., 2015; Oldenburg and Sabatini, 2015).

On the other hand, the STN receives direct cortical inputs and projects to the $\mathrm{SNr} / \mathrm{GPi}$ and $\mathrm{GPe}$, and thus forms the cortico-STN-SNr/GPi hyperdirect pathway (Fig. $1 A$; Nambu et al., $1996,2000,2002)$. The hyperdirect pathway rapidly conveys cortical activity to the $\mathrm{SNr} / \mathrm{GPi}$, which may reset the cortical activity related to ongoing movements. However, the functions of the hyperdirect pathway remain to be fully elucidated. Our previous study successfully eliminated cortico-STN neurons originating from the supplementary motor area of the primate brain by combining a neuron-specific retrograde gene-transfer vector and immunotoxin-mediated tract targeting (Inoue et al., 2012). However, these animals did not show any apparent motor deficits by simple neurologic observations. In the present study, we used mice whose motor activity could be quantitatively measured. In addition, to selectively eliminate the cortico-STN projection, we used a photodynamic technique (PDT; Madison et al., 1990; Macklis, 1993; Madison and Macklis, 1993), which did not require viral vectors. The PDT successfully eliminated corticocortical and corticothalamic neurons in mice or rats (Macklis, 1993; Madison and Macklis, 1993; Magavi et al., 2000) and cats (Eyding et al., 2003), and affected vocal behaviors in songbirds (Scharff et al., 2000). We observed basal ganglia activity and motor behaviors of mice in awake conditions before and after cortico-STN elimination and found that its elimination reduced cortically evoked early excitation in the GPe and $\mathrm{SNr}$, and induced locomotor hyperactivity.

\section{Materials and Methods}

Animals. Male C57BL/6 mice aged 12-16 weeks at the start of the experiment were housed two to three per cage and were used in the present study. The experimental protocol was approved by the Institutional Animal Care and Use Committee of the National Institutes of Natural
Sciences, and all procedures were conducted according to the guidelines of the National Institutes of Health Guide for the Care and Use of Laboratory Animals.

Experimental design. The following experiments were performed (Fig. 1A). (1) Histologic examinations were performed to confirm selective elimination of cortical neurons projecting to the STN (five mice were used). RetroBeads conjugated with chlorin e6 (RetroBeads-chlorin) were injected into the unilateral (right) STN. After 2 weeks, the right motor cortex was irradiated with a near-infrared laser. One month after laser irradiation, injection sites in the STN and cortical neurons projecting to the STN were examined histologically. (2) Electrophysiological recordings were performed to examine the effects of cortico-STN elimination on GPe and SNr activity (five mice; three for GPe, five for SNr). RetroBeads-chlorin were injected into the unilateral (right) STN. Two weeks after RetroBeads injection, recordings of spontaneous activity and cortically evoked responses in the right GPe and $\mathrm{SNr}$ were started and performed for 2 weeks ("before" cortico-STN elimination). Four weeks after RetroBeads injection, the right motor cortex was irradiated with a near-infrared laser. One month after laser irradiation, neuronal recordings in the GPe and SNr were resumed in the same five mice ("after" cortico-STN elimination). Recording sites were reconstructed based on histologic examination. (3a) Behavioral tests were performed to assess locomotor activity after bilateral elimination of the cortico-STN projection (10 mice). Ten mice were randomly divided into two groups. In the first group (five mice), RetroBeads-chlorin were injected into the bilateral STN ("cortico-STN eliminated" mice), while in the second group (the other five mice), RetroBeads without chlorin e6 (RetroBeads w/o chlorin) were injected into the bilateral STN ("control" mice). One week after RetroBeads injection, locomotor activity was measured and continued for $7 \mathrm{~d}$ ("before" laser irradiation). Two weeks after RetroBeads injection, the bilateral motor cortices of both groups were irradiated with a near-infrared laser. Then, locomotor activity was chronologically measured for 4 weeks ("after" laser irradiation). (3b) Behavioral tests were performed to assess rotational behaviors after unilateral elimination of the cortico-STN projection (five mice). RetroBeads-chlorin were injected into the unilateral (right) STN. One week after RetroBeads injection, rotational behaviors were observed ("before" cortico-STN elimination). Apomorphine was also systemically administrated. Two weeks after RetroBeads injection, the right motor cortex was irradiated 
with a near-infrared laser. One month after laser irradiation, rotational behaviors were observed without and with apomorphine administration ("after" cortico-STN elimination). Injection sites of RetroBeads in the STN and cortical neurons projecting to the STN were also examined histologically in experiments 2, 3a, and 3b. Details of the method are described below.

Surgery. To painlessly fix the head of an awake mouse to the stereotaxic apparatus, a small U-frame head holder was first mounted on its head as previously described (Chiken et al., 2008, 2015; Sano et al., 2013; Dwi Wahyu et al., 2021). Briefly, each mouse was anesthetized with ketamine hydrochloride $(100 \mathrm{mg} / \mathrm{kg}$ body weight, i.p.) and xylazine hydrochloride $(5 \mathrm{mg} / \mathrm{kg}$, i.p.) and then fixed in the stereotaxic apparatus. After widely exposing the skull, the periosteum and blood on the skull were completely removed. The exposed skull was completely covered with bone-adhesive resin (Bistite II, Tokuyama Dental) and acrylic resin (Unifast II, GC Corporation). A small polyacetal U-frame head holder for head fixation was mounted on the head and fixed with acrylic resin.

After recovery from the first surgery (2-3 d later), the mouse was positioned in a stereotaxic apparatus with its head restrained using the U-frame head holder under anesthesia with ketamine hydrochloride $(50-100 \mathrm{mg} / \mathrm{kg}$, i.p.). Part of the skull was removed to enable access to the motor cortex, STN, GPe, and SNr. A pair of stimulating electrodes (tip distance, $300-400 \mu \mathrm{m}$ ) made of $50-\mu \mathrm{m}$-diameter Teflon-coated tungsten wires (California Fine Wire) was inserted into the caudal forelimb area of the motor cortex $(0.8 \mathrm{~mm}$ anterior to bregma and $1.7 \mathrm{~mm}$ lateral to bregma), which was confirmed by forelimb movements evoked by intracortical microstimulation (train of 10 pulses at $333 \mathrm{~Hz}, 200 \mu$ s in duration, up to $30 \mu \mathrm{A}$ in strength). Stimulating electrodes were then fixed in place using acrylic resin. The cortex around the insertion sites remained uncovered for later laser irradiation.

Injection of RetroBeads-chlorin into the STN. To selectively eliminate the cortico-STN projection, we used a PDT with chlorin e6 (Madison et al., 1990; Macklis, 1993; Madison and Macklis, 1993; Sheen and Macklis, 1994; Magavi et al., 2000; Scharff et al., 2000; Eyding et al., 2003). We first localized the STN by recording the neuronal activity using similar methods described in "Electrophysiological recordings of GPe and $\mathrm{SNr}$ activity" section below. A glass-coated Elgiloy-alloy microelectrode $(\sim 1.0 \mathrm{M} \Omega)$ was inserted vertically into the STN $(1.8-2.2 \mathrm{~mm}$ posterior to bregma and $1.3-1.8 \mathrm{~mm}$ lateral to bregma; Paxinos and Franklin, 2001). STN neurons were characterized by moderate firing frequency $(\sim 40 \mathrm{~Hz})$ and a biphasic excitatory response composed of early and late excitation to the ipsilateral cortical stimulation (Nambu et al., 2000; Iwamuro et al., 2017; Polyakova et al., 2020).

RetroBeads-chlorin were made by conjugating retrogradely transportable microspheres (rhodamine-labeled RetroBeads, Lumafluor) with chlorin e6, a photosensitizer (Frontier Scientific), using a previously described method (Madison and Macklis, 1993). A glass micropipette with a Teflon-coated tungsten wire (diameter, $30 \mu \mathrm{m}$ ) for recordings was connected to a Hamilton microsyringe and filled with RetroBeadschlorin. Based on the results of the STN mapping, a glass micropipette was inserted vertically into the center of the STN. After electrophysiological recordings confirmed that the tip of the glass pipette was located inside the forelimb region of the STN, RetroBeads-chlorin were slowly injected (Fig. 1A; one site, $200 \mathrm{nl}, 20 \mathrm{nl} / \mathrm{min}$ ) by using a microsyringe pump (UltraMicroPump II and Micro4, WPI). After injection, the glass micropipette was left in place for $5 \mathrm{~min}$. RetroBeads diffused only within a restricted region because of their relatively large sizes (Macklis and Quattrochi, 1991). RetroBeads would be retrogradely transported to the somata of the cortical neurons projecting to the STN in 2 weeks and persist there (Fig. 1A; Madison et al., 1990), suggesting that anterograde and transsynaptic transports were negligible.

Electrophysiological recordings of GPe and SNr activity (Fig. 1A). Each mouse was placed in a stereotaxic apparatus with its head painlessly restrained using the U-frame head holder in awake conditions. A glasscoated Elgiloy-alloy microelectrode $(\sim 1.0 \mathrm{M} \Omega$ at $1 \mathrm{kHz})$ was inserted vertically into the somatomotor areas of the GPe $(0.3-0.7 \mathrm{~mm}$ posterior to bregma and 1.4-2.2 mm lateral to bregma; Paxinos and Franklin, 2001) and $\mathrm{SNr}$ (3.0-3.4 $\mathrm{mm}$ posterior to bregma and 1.0-1.8 mm lateral to bregma) through the dura mater using a hydraulic microdrive
(Narishige Scientific Instrument). Signals from the electrode were amplified, filtered $(0.2-5 \mathrm{kHz})$, and continuously monitored using an oscilloscope. The GPe and $\mathrm{SNr}$ were identified by the depth profile of the microelectrode penetrations and their sustained high-frequency spontaneous firings. The activity of GPe and SNr neurons was isolated, and the action potentials were converted to digital pulses using a homemade time-amplitude window discriminator, and sampled at $2 \mathrm{kHz}$ by using LabVIEW software (National Instruments) and a computer. Responses to the cortical stimulation (bipolar stimulation, single monophasic pulse at $0.7 \mathrm{~Hz}, 200 \mu \mathrm{s}$ in duration, and $20-50 \mu \mathrm{A}$ strength) through the electrodes implanted in the forelimb area of the right motor cortex were recorded and analyzed by constructing peristimulus time histograms (PSTHs; bin width, $1 \mathrm{~ms}$; summed for 100 stimulus trials). The spontaneous firings of GPe and $\mathrm{SNr}$ neurons were also recorded as digitized spikes for $50 \mathrm{~s}$. Neuronal recordings were performed while the mice were awake but not moving their limbs to avoid the effects of movements on neuronal activity. In the final experiment, several neuronal recording sites were marked by passing cathodal direct current $(20 \mu \mathrm{A}$ for $30 \mathrm{~s}$ ) through the recording electrode.

Laser irradiation on the motor cortex. After RetroBeads injection, the motor cortex (1.35-mm-diameter area centered at the insertion points of the cortical stimulating electrodes) was irradiated with a near-infrared laser $(670 \mathrm{~nm}, 2.4 \mathrm{~mW}$; Shäfter + Kirchhoff) for $15 \mathrm{~min}$ (Fig. 1A). Chlorin e6 was activated by the laser and generated reactive oxygen species exclusively in the labeled neurons (Madison et al., 1990; Macklis, 1993; Madison and Macklis, 1993; Sheen and Macklis, 1994). Reactive oxygen species induced a slow (several weeks) apoptotic process (Sheen and Macklis, 1994), and the motor cortical neurons projecting to the STN were selectively eliminated within 1 month. This technique can induce the pathway-selective targeted cell death with other pathways intact; callosal-projecting neurons within layers $2 / 3$ and 5 (Macklis, 1993; Madison and Macklis, 1993), and layer 6 feedback-projecting neurons from the primary visual cortex to the lateral geniculate nucleus (Eyding et al., 2003) were selectively eliminated; laser irradiation alone or RetroBeads-chlorin injection alone caused no cellular injury (Madison and Macklis, 1993), and laser irradiation on RetroBeadschlorin induced reactive oxygen, but laser irradiation on RetroBeads w/o chlorin did not (Sheen and Macklis, 1994).

Behavioral tests to assess locomotor activity. Each mouse was placed in a $30-\mathrm{cm}$-diameter arena and video recorded for $10 \mathrm{~min}$ using a digital video camera. Distances traveled for $10 \mathrm{~min}$ were calculated using a computer. Locomotor activity was measured for $7 \mathrm{~d}$ before laser irradiation, and the mean value was calculated in each mouse and used as the baseline activity. We confirmed no significant activity difference between control and cortico-STN-eliminated groups before laser irradiation. Locomotor activity was chronologically measured after laser irradiation and indicated by the ratio to the baseline activity in each mouse.

Behavioral tests to assess rotational behaviors. Each mouse was placed in a $30-\mathrm{cm}$-diameter arena and video recorded for $30 \mathrm{~min}$ using a digital video camera. The numbers of ipsilateral (to the cortico-STN eliminated right side) and contralateral rotations in $30 \mathrm{~min}$ were counted using a computer. On a different day, a nonselective dopamine agonist, apomorphine (Sigma-Aldrich), was systemically administered (before cortico-STN elimination, $3.0 \mathrm{mg} / \mathrm{kg}$, i.p.; after cortico-STN elimination, $0.5,1.5,3.0$, and $5.0 \mathrm{mg} / \mathrm{kg}$, i.p.) $5 \mathrm{~min}$ before testing to enhance basal ganglia activity and reveal subtle activity asymmetry between the left and right basal ganglia (Delfs et al., 1995; Waszczak et al., 2002; Sano et al., 2003). The ratios of the ipsilateral rotations to the total rotations (sum of ipsilateral and contralateral rotations) were calculated.

Histology. The mice were deeply anesthetized with sodium pentobar-

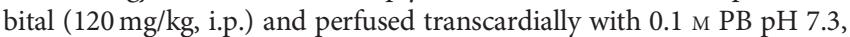
followed by $10 \%$ formalin in $0.1 \mathrm{M} \mathrm{PB}$, and then $0.1 \mathrm{M}$ PB containing $10 \%$ sucrose. Brains were immediately removed, immersed in $0.1 \mathrm{M} \mathrm{PB}$ containing $30 \%$ sucrose, and then cut into $50-\mu \mathrm{m}$-thick coronal sections on a freezing microtome. The sections were permeabilized in PBS $\mathrm{pH}$ 7.2 containing $0.1 \%$ Triton X-100, incubated with NeuroTrace (dilution factor, 200; Thermo Fisher Scientific), mounted onto glass slides, and coverslipped. The sections were observed under a fluorescence microscope. Retrogradely transported RetroBeads were detected with red 
fluorescent rhodamine, and neuronal somata were visualized with green fluorescent NeuroTrace. The numbers of cells double-labeled with RetroBeads and NeuroTrace in layer 5 of the motor cortex were counted. To localize the electrophysiological recording sites, the sections were mounted onto gelatin-coated glass slides, stained with $0.5 \%$ cresyl violet, dehydrated, and coverslipped. The sections were observed under a light microscope, and the recording sites were reconstructed according to the lesions made by the current injection and the traces of the electrode tracks. Stimulation sites in the motor cortex were also verified histologically.

Data analysis. The responses of GPe and SNr neurons to the cortical stimulation were assessed by PSTHs using Igor Pro software (WaveMetrics). The mean and SD of the firing rate during the $100 \mathrm{~ms}$ preceding the onset of stimulation were calculated from a PSTH and were considered to be the values for the baseline discharge. Changes in the firing rate in response to the cortical stimulation (i.e., excitation or inhibition) were judged to be significant if the firing rate during at least two consecutive bins $(2 \mathrm{~ms}$ ) reached the statistical level of $p<0.05$ (onetailed $t$ test), when compared with the baseline discharge (Chiken et al., 2008, 2015; Sano et al., 2013). The latency of each response component was defined as the time of the first bin that exceeded this level. Responses were judged to end when two consecutive bins fell below the significance level. The end point was determined as the time of the last bin that exceeded this level. The duration of each response component was defined as the time during the significant change (between the latency and the end point). The amplitude of each response component was defined as the number of spikes that occurred during the significant change minus that of the baseline discharge (i.e., the area of the response); positive or negative values indicate excitatory or inhibitory responses. If no significant changes were found, the amplitude and duration were set to zero. For population PSTHs, PSTHs of all responsive neurons were averaged and then smoothed using a Gaussian window ( $\sigma=1.0 \mathrm{~ms})$.

The spontaneous firing rates and the parameters that characterized the firing patterns were calculated from digitized recordings for 50 s (Hutchinson et al., 1997; Chiken et al., 2008, 2015; Sano et al., 2013; Dwi Wahyu et al., 2021), namely, the mean, coefficient of variation (CV), skewness, and kurtosis of interspike intervals (ISIs); burst index (a ratio of the mean ISI to the mode ISI); and the percentage of spikes in bursts detected by the Poisson surprise method (Poisson surprise value, $-\log _{10} p \geq 3.0$; spikes/burst $\geq 3$; Legéndy and Salcman, 1985). Autocorrelograms (bin width, $0.5 \mathrm{~ms}$ ) were constructed from the same continuous digitized recordings for $50 \mathrm{~s}$.

Statistical analyses. Statistical analyses were performed using Prism software (GraphPad). The numbers of double-labeled cells in the laserirradiated and surrounding nonirradiated cortices were compared by using two-tailed paired $t$ tests. The parameters of cortically evoked response components and spontaneous neuronal activity before and after cortico-STN elimination were compared by using two-tailed $t$ tests. The proportions of cortically evoked response patterns before and after cortico-STN elimination were examined by using $\chi^{2}$ tests. Behavioral data were analyzed by using two-way repeated-measures ANOVA with Bonferroni's test, one-way repeated-measures ANOVA with Tukey's test, or two-tailed paired $t$ tests. All values are expressed as the mean \pm $\mathrm{SD}$, unless otherwise stated.

\section{Results}

\section{Selective elimination of cortical neurons projecting to the STN}

We injected rhodamine-labeled RetroBeads-chlorin into the STN and found that they were successfully localized within the STN (Fig. 1B1,B2). One month after laser irradiation, rhodamine-labeled layer 5 neurons projecting to the STN were selectively eliminated in the laser-irradiated cortex (Fig. 1C1,C2,D1), while they were spared from elimination in the surrounding nonirradiated cortex (Fig. 1C1,C2,D2). Approximately $67 \%$ of labeled cells were eliminated in the laser-irradiated cortex compared with the surrounding nonirradiated cortex (Fig. 1D3; $t_{(4)}=5.17, p=0.007$, two-tailed paired $t$ test). Nonlabeled neurons in the laser-irradiated cortex remained intact (Fig. 1D1). Only large neurons in layer 5 were labeled (Fig. 1C1,C2,D2), and no labeled terminals were found in the $\mathrm{SNr}$ (data not shown), suggesting that transsynaptic and anterograde transports of RetroBeads were negligible.

\section{GPe and SNr activity before and after cortico-STN elimination}

We recorded the activity of GPe and $\mathrm{SNr}$ neurons in response to the stimulation of the forelimb area of the motor cortex in awake conditions and constructed PSTHs (Fig. 2). Before cortico-STN elimination, a triphasic response composed of early excitation, followed by inhibition and late excitation (Fig. 2A1,B1, Before) was the most common in both GPe (41\%) and $\mathrm{SNr}(37 \%)$ neurons (Fig. 2A2,B2, Before). After cortico-STN elimination, drastic changes were observed in the response patterns; early excitation was diminished in both GPe and SNr neurons (Fig. $2 A 1, B 1$, After). The numbers of neurons with early excitation, such as a triphasic response, excitation-inhibition, excitation-excitation, and early excitation, were significantly decreased in the GPe (before, 73\%; after, 23\%; $\chi^{2}=30.7, \mathrm{df}=1, p<0.001,1-\beta$ $=0.994, \chi^{2}$ test; Fig. $2 A 2$, cold colors) and SNr (before, $81 \%$; after, $26 \% ; \chi^{2}=33.5, \mathrm{df}=1, p<0.001,1-\beta=0.999$; Fig. $2 B 2$, cold colors), and the responses without early excitation dominated (Fig. 2A2,B2, After, warm colors), such as inhibition-excitation (41\%) in the GPe, and monophasic inhibition (30\%) and late excitation (31\%) in the SNr. These changes were also observed in the population PSTHs; early excitation was greatly diminished in both GPe and $\mathrm{SNr}$ neurons after cortico-STN elimination (Fig. 2A3,B3). Quantitative analyses (Table 1) showed that the duration and amplitude of early excitation in the GPe (duration, $t_{(124)}=4.95$, $p<0.001$; amplitude, $t_{(124)}=5.13, p<0.001$; two-tailed $t$ test $)$ and $\mathrm{SNr}$ (duration, $t_{(106)}=4.28, p<0.001$; amplitude, $t_{(106)}=4.33$, $p<0.001)$ were significantly decreased after cortico-STN elimination. On the other hand, cortically evoked inhibition and late excitation exhibited little change after cortico-STN elimination (Fig. 2A1,A3,B1,B3, Table 1). These observations indicate that the cortico-STN projection conveys cortical excitation to the GPe and SNr with a short latency.

We additionally examined spontaneous activity of GPe and SNr neurons before and after cortico-STN elimination (Fig. 3, Table 2) because their changes in firing rates and patterns are frequently suggested in movement disorders (Wichmann et al., 2011). Before cortico-STN elimination, GPe and SNr neurons fired randomly at high frequency (Fig. $3 A, B$, Before, Table 2) as reported previously (Chiken et al., 2008, 2015; Sano et al., 2013; Dwi Wahyu et al., 2021). After cortico-STN elimination, however, no significant changes were observed in firing rates, autocorrelograms, and parameters characterizing firing patterns, such as CV, skewness, and kurtosis of ISIs; burst index; and the percentage of spikes in bursts (Fig. $3 A, B$, After, Table 2).

\section{Locations of recorded GPe and SNr neurons}

The locations of recorded GPe and $\mathrm{SNr}$ neurons are plotted using different colors based on cortically evoked response patterns in Figure 4. We mainly recorded in the middle and lateral parts of the GPe and the dorsolateral part of the SNr, which correspond to the somatomotor regions (Chiken et al., 2008, 2015; Sano et al., 2013; Dwi Wahyu et al., 2021). Before cortico-STN elimination, the neurons with early excitation dominated in both 

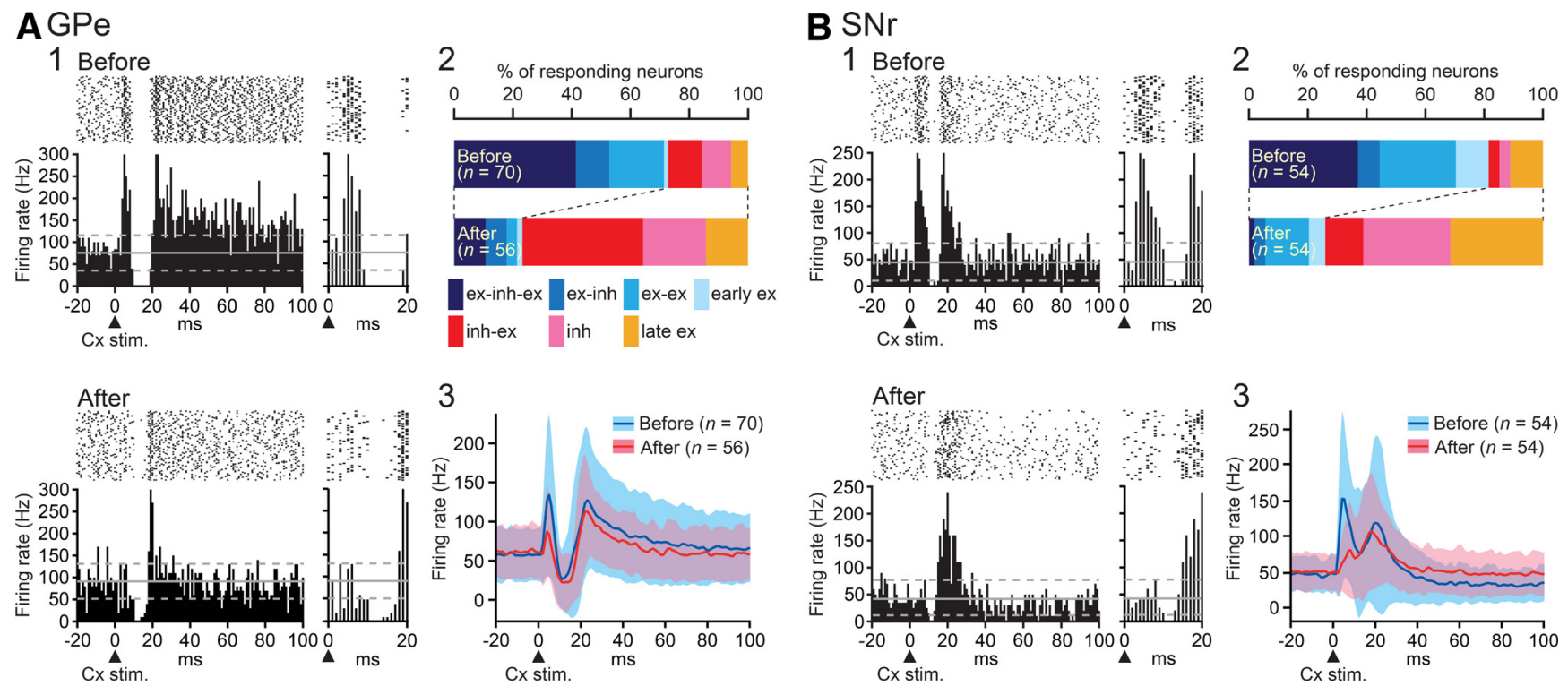

Figure 2. $\boldsymbol{A}, \boldsymbol{B}$, Diminished cortically evoked early excitation in the GPe $(\boldsymbol{A})$ and SNr $(\boldsymbol{B})$ after cortico-STN elimination. $\boldsymbol{A}$ 1, $\boldsymbol{B} 1$, Raster plots and PSTHs of typical GPe $(\boldsymbol{A} 1)$ and SNr $(\boldsymbol{B} 1)$ neurons before (Before) and after (After) cortico-STN elimination. Cortical stimulation ( $\mathrm{X}$ stim.) was applied at $0 \mathrm{~ms}$. Raster plots and PSTHs expanded between 0 and $20 \mathrm{~ms}$ are also shown. The mean firing rate of the baseline discharge and statistical levels of $p<0.05$ (one-tailed $t$ test) are indicated by a gray solid line and gray dashed lines, respectively. A2, $\boldsymbol{B 2}$, Response patterns of GPe (A2) and SNr (B2) neurons before and after cortico-STN elimination (n, number of neurons sampled). A3, B3, Population PSTHs of GPe (A3) and SNr (B3) neurons before (blue) and after (red) cortico-STN elimination. The shaded areas represent \pm SD. See also Table 1.

Table 1. Cortically evoked responses of GPe and SNr neurons before and after cortico-STN elimination

\begin{tabular}{|c|c|c|c|c|}
\hline & \multicolumn{2}{|l|}{ GPe } & \multicolumn{2}{|l|}{$\mathrm{SNr}$} \\
\hline & Before & After & Before & After \\
\hline Neurons recorded, $N$ & 118 & 100 & 83 & 91 \\
\hline \multicolumn{5}{|l|}{ Early excitation } \\
\hline Latency (ms) & $3.9 \pm 1.4$ & $4.0 \pm 1.3$ & $4.5 \pm 2.5$ & $7.3 \pm 2.6^{* *}$ \\
\hline Duration (ms) & $2.0 \pm 1.8$ & $0.5 \pm 1.2^{* * *}$ & $3.1 \pm 3.0$ & $0.9 \pm 2.1^{* * *}$ \\
\hline Latency (ms) & $9.5 \pm 1.9$ & $9.6 \pm 2.4$ & $11.7 \pm 4.1$ & $11.3 \pm 3.7$ \\
\hline Duration (ms) & $5.9 \pm 5.2$ & $5.3 \pm 4.0$ & $1.9 \pm 3.2$ & $1.4 \pm 2.3$ \\
\hline Amplitude (spikes) & $-38.5 \pm 37.9$ & $-34.1 \pm 30.7$ & $-6.6 \pm 16.7$ & $-7.0 \pm 11.9$ \\
\hline \multicolumn{5}{|l|}{ Late excitation } \\
\hline Latency (ms) & $18.2 \pm 5.0$ & $19.3 \pm 3.1$ & $15.7 \pm 4.9$ & $14.6 \pm 5.3$ \\
\hline Duration (ms) & $13.5 \pm 17.2$ & $9.7 \pm 8.4$ & $8.7 \pm 7.5$ & $10.0 \pm 9.5$ \\
\hline
\end{tabular}

Values are the mean $\pm S D$.

${ }^{* *} p<0.01,{ }^{* * *} p<0.001$, significantly different from the values before cortico-STN elimination (two-tailed $t$ test).

the GPe and SNr (Fig. 4A,B, Before, cold colors). After corticoSTN elimination, however, the neurons without early excitation dominated and were distributed in the same areas of the GPe and $\mathrm{SNr}$ (Fig. 4A,B, After, warm colors).

\section{Motor hyperactivity after cortico-STN elimination}

Next, we examined locomotor activity of mice before and after bilateral cortico-STN elimination (Fig. 5A). There was a gradual increase in the locomotor activity of cortico-STN eliminated mice starting 1 week after laser irradiation, while that of control mice remained unchanged. The gradual increase may reflect a slow elimination process of cortico-STN neurons. The activity level became significantly higher than that of control mice in $2-4$ weeks $\left(F_{(7,28)}=4.4, p=0.0016,1-\beta=0.99\right.$ for treatment $\times$ day interaction, two-way repeated-measures ANOVA; day 14, $p=0.045$; day 21, $p=0.016$; day 28, $p=0.0003$, Bonferroni's test; Fig. $5 A$ ).

On the other hand, unilateral cortico-STN elimination induced no apparent changes in locomotion (Fig. 5B). However, apomorphine injection $(1.5,3.0$, and $5.0 \mathrm{mg} / \mathrm{kg})$ induced ipsilateral rotations to the cortico-STN-eliminated side in a dose-dependent manner $\left(F_{(4,20)}=20.2, p<0.001,1-\beta=0.999\right.$, oneway repeated-measures ANOVA; $1.5,3.0$, and $5.0 \mathrm{mg} / \mathrm{kg}$, $p<0.001$, Tukey's test; Fig. 5B). Apomorphine injection $(3.0 \mathrm{mg} / \mathrm{kg})$ did not induce changes in rotations before corticoSTN elimination, but it induced ipsilateral rotations after cortico-STN elimination $\left(t_{(4)}=8.83, p<0.001\right.$, two-tailed paired $t$ test).

These observations indicate that cortico-STN elimination induced motor hyperactivity at the behavioral level. Other than 


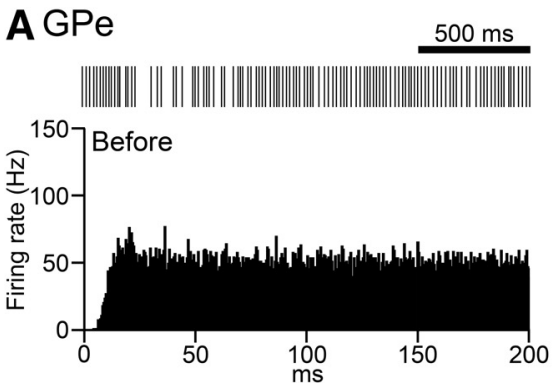

\section{B SNr}
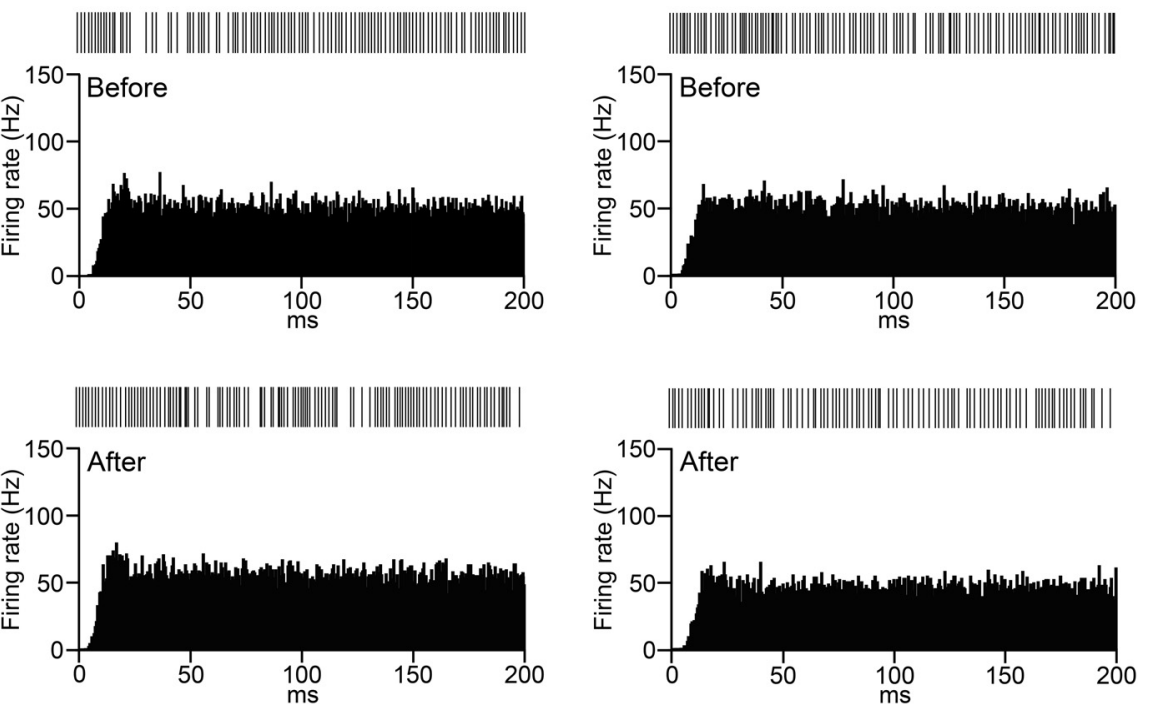

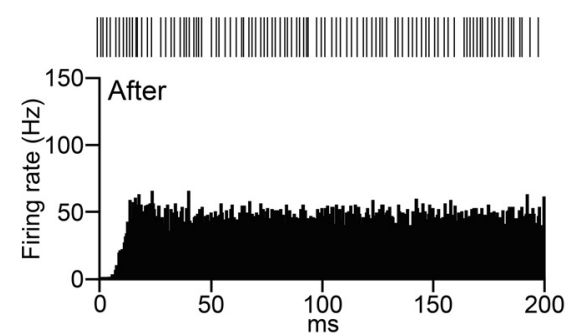

Figure 3. $\boldsymbol{A}, \boldsymbol{B}$, Spontaneous activity of typical GPe $(\boldsymbol{A})$ and $\mathrm{SNr}(\boldsymbol{B})$ neurons before (Before) and after (After) cortico-STN elimination, which is denoted by the digitized spikes and autocorrelograms (bin width, $0.5 \mathrm{~ms}$ ). See also Table 2.

al., 2017; Ozaki et al., 2017; Dwi Wahyu et al., 2021). After elimination of the cortico-STN projection, early excitation was diminished in both GPe and SNr neurons, which is also consistent with previous reports that elimination of the corticoSTN projection, blockade of the corticoSTN neurotransmission, and activity blockade or lesions of the STN diminished early excitation in the GPe/SNr/GPi (Ryan and Clark, 1991; Maurice et al., 1999; Nambu et al., 2000; Inoue et al., 2012). The present results have added another strong piece of evidence that the cortico-STN projection mediates early excitation in the GPe/SNr/GPi. On the other hand, cortically evoked inhibition and late excitation exhibited little change after cortico-STN elimination, suggesting that the contribution of the cortico-STN projection to inhibition and late excitation is minor. Actually, inhibition and late excitation in the GPe/SNr/GPi have been shown to be mediated by the corticostriato-GPe/SNr/GPi and cortico-striato-

Table 2. Firing rates and patterns of GPe and SNr neuron before and after cortico-STN elimination

\begin{tabular}{lccccc}
\hline & GPe & & \multicolumn{2}{l}{ SNr } \\
\cline { 2 - 3 } & Before & After & & Before & After \\
\hline Neurons $(n)$ & 70 & 56 & 54 & 54 \\
Firing rate $(\mathrm{Hz})$ & $52.3 \pm 26.6$ & $53.9 \pm 20.4$ & $47.7 \pm 16.8$ & $50.9 \pm 13.7$ \\
ISI mean (ms) & $27.4 \pm 22.6$ & $23.7 \pm 19.2$ & & $28.0 \pm 11.0$ & $24.4 \pm 6.4$ \\
$\quad$ CV & $1.0 \pm 0.6$ & $0.8 \pm 0.4$ & $0.8 \pm 0.2$ & $0.9 \pm 0.3$ \\
$\quad$ Skewness & $4.5 \pm 2.6$ & $3.4 \pm 1.9$ & $3.6 \pm 2.3$ & $2.9 \pm 0.7$ \\
$\quad$ Kurtosis & $41.1 \pm 53.9$ & $26.8 \pm 39.1$ & & $34.5 \pm 53.0$ & $18.2 \pm 6.6$ \\
Burst index & $3.6 \pm 4.7$ & $2.3 \pm 2.1$ & $3.2 \pm 2.9$ & $3.7 \pm 1.4$ \\
Spikes in bursts (\%) & $8.1 \pm 17.0$ & $4.7 \pm 8.4$ & $3.4 \pm 5.7$ & $4.8 \pm 4.8$
\end{tabular}

Values are the mean \pm SD. No significant changes were observed between before and after cortico-STN elimination ( $p>0.05$, two-tailed $t$ test).

increased locomotor activity and apomorphine-induced ipsilateral rotations, we did not observe any other apparent abnormal behaviors or motor deficits in cortico-STN-eliminated mice.

\section{Discussion}

Here, we investigated the functions of the cortico-STN hyperdirect pathway. We selectively eliminated the cortico-STN projection and observed neuronal activity and motor behaviors. After cortico-STN elimination, cortically evoked early excitation in the $\mathrm{GPe}$ and $\mathrm{SNr}$ was diminished, and motor hyperactivity was observed; locomotor activity was increased, and apomorphineinduced ipsilateral rotations were induced. Our data suggest that the cortico-STN hyperdirect pathway quickly conveys cortical excitation to the $\mathrm{SNr}$, and resets or suppresses cortical activity and movements.

\section{GPe and SNr activity mediated by the cortico-STN projection}

In control mice, cortical stimulation induced a triphasic response composed of early excitation, followed by inhibition and late excitation in the GPe and $\mathrm{SNr}$, which is consistent with previous reports (Ryan and Clark, 1991; Maurice et al., 1999; Nambu et al., 2000; Chiken et al., 2008, 2015; Sano et al., 2013; Iwamuro et
GPe-STN-GPe/SNr/GPi pathways, respectively (Kita et al., 2004; Tachibana et al., 2008; Sano et al., 2013).

Previous studies have reported that lesions or inactivation of the STN reduced firing rates and changed firing patterns of GPe and GPi neurons (Hamada and DeLong, 1992; Vitek et al., 1999; Nambu et al., 2000). The present study showed, however, that elimination of the cortico-STN projection induced neither reduction of spontaneous firing rates nor changes of firing patterns in the GPe and SNr, which is consistent with the findings of our previous report (Inoue et al., 2012). These results suggest that STN neurons maintain their spontaneous activity at least to some extent after cortico-STN elimination. Although STN activity was not recorded after cortico-STN elimination in the present study, previous studies have shown that STN neurons are autonomously active without any cortical inputs (Nakanishi et al., 1987; Bevan and Wilson, 1999; Do and Bean, 2003; Polyakova et al., 2020) and can maintain the firing rates and patterns of GPe/ $\mathrm{SNr} / \mathrm{GPi}$ neurons through the STN-GPe/SNr/GPi excitatory projection (Kita et al., 2004; Tachibana et al., 2008).

Could cortically evoked responses in the GPe/SNr be affected by motor hyperactivity after cortico-STN elimination? This is unlikely because (1) neuronal recordings were performed while the mice were not moving their limbs, and (2) motor hyperactivity after unilateral cortico-STN elimination was subtle and detectable only after apomorphine administration.

\section{Motor suppression by the cortico-STN projection}

The current results strongly support the idea that the hyperdirect pathway conveys fast excitatory signals from the motor cortex to the $\mathrm{SNr} / \mathrm{GPi}$, inhibits thalamic activity, and resets or suppresses the cortical activity related to ongoing movements (Mink and Thach, 1993; Nambu et al., 2000, 2002; Leblois et al., 2006; Guthrie et al., 2013). Imaging and electrophysiological studies in humans, rats, and monkeys have reported that STN neurons were activated during stopping movements in reaction to a stop signal or switching movements (Isoda and Hikosaka, 2008; Jahfari et al., 2011; Alegre et al., 2013; Schmidt et al., 2013; Pasquereau and Turner, 2017). Manipulating the activity of the 
A GPe
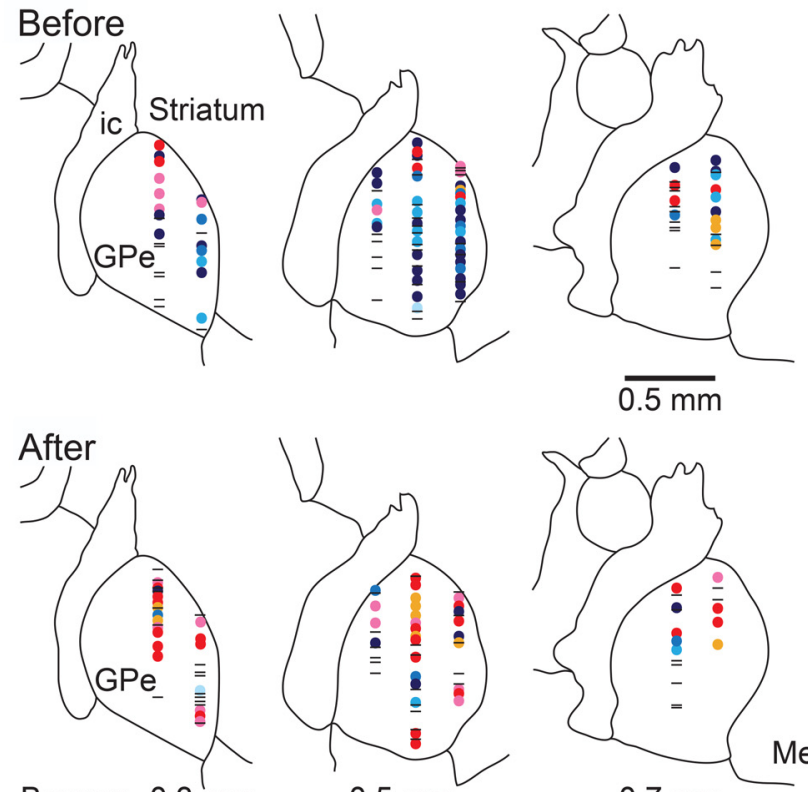

Bregma $-0.3 \mathrm{~mm}$

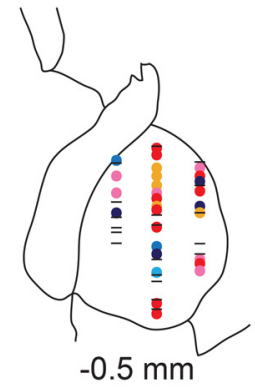

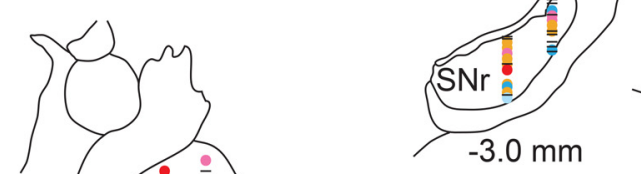
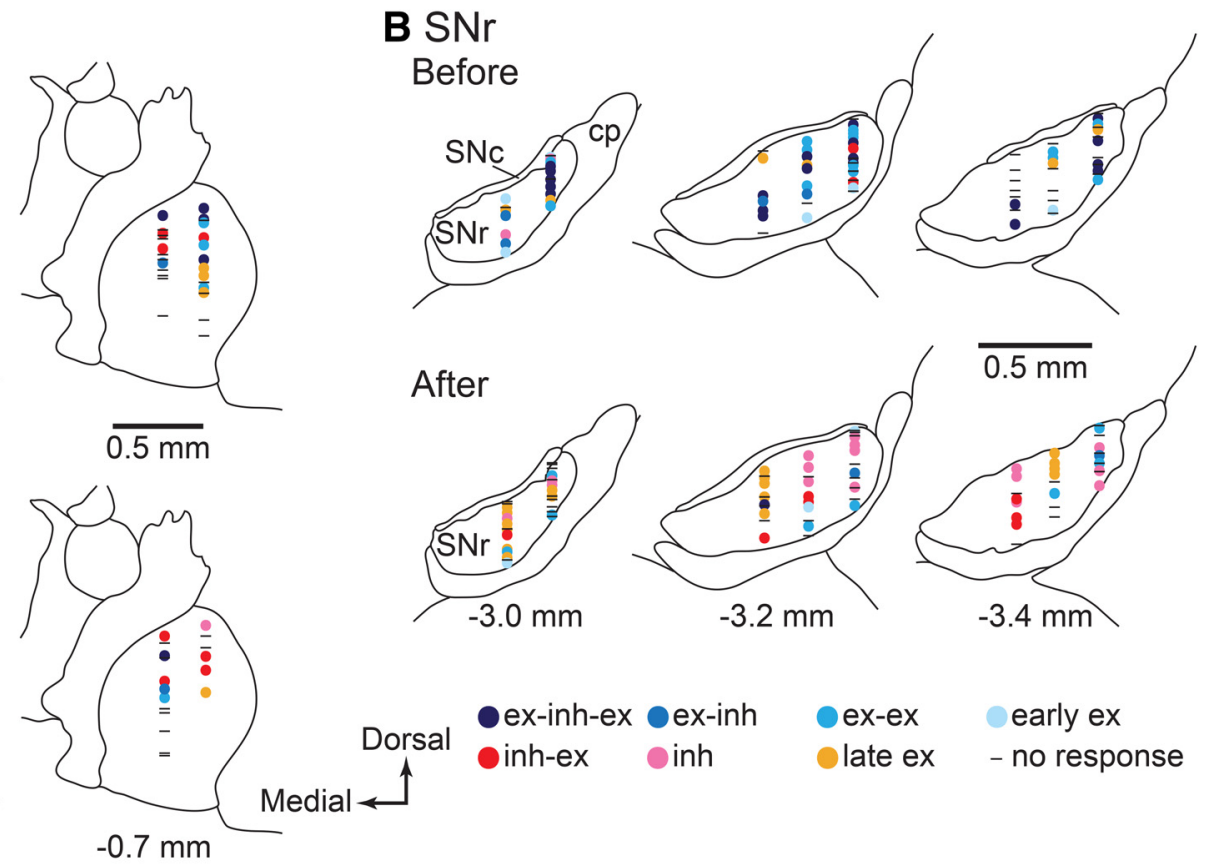

ex-ex

early ex

late ex

- no response

Figure 4. $\quad \boldsymbol{A}, \boldsymbol{B}$, Recording sites in the GPe (A) and SNr (B) before (Before) and after (After) cortico-STN elimination. Data from all mice (GPe, 3 mice; SNr, 5 mice) are overlaid on the coronal sections. Locations of recorded neurons are indicated by different colors based on response patterns evoked by the cortical stimulation. Numbers below the sections represent the distances from bregma. cp, cerebral peduncle; ic, internal capsule.

A

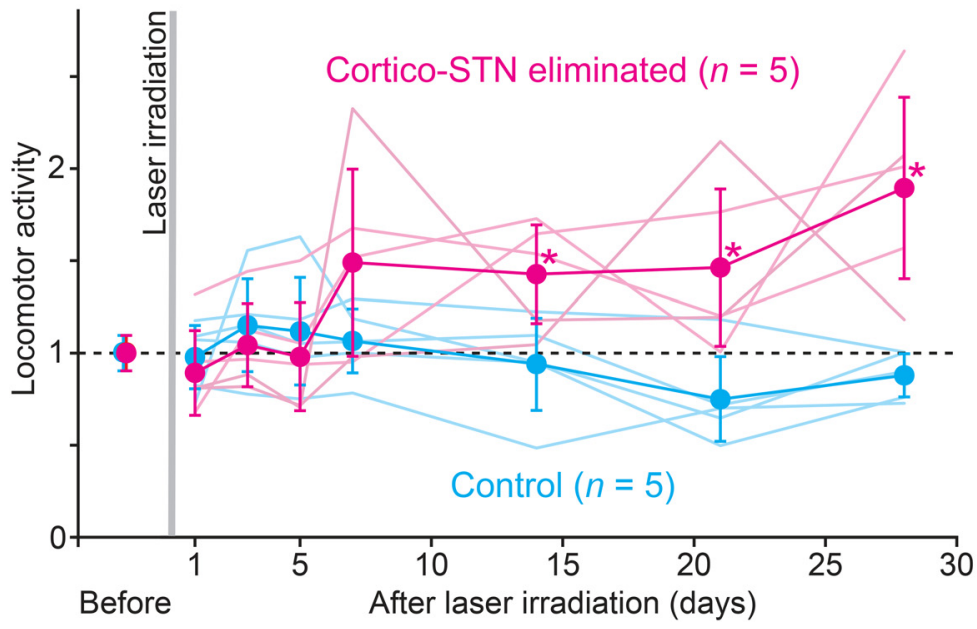

B

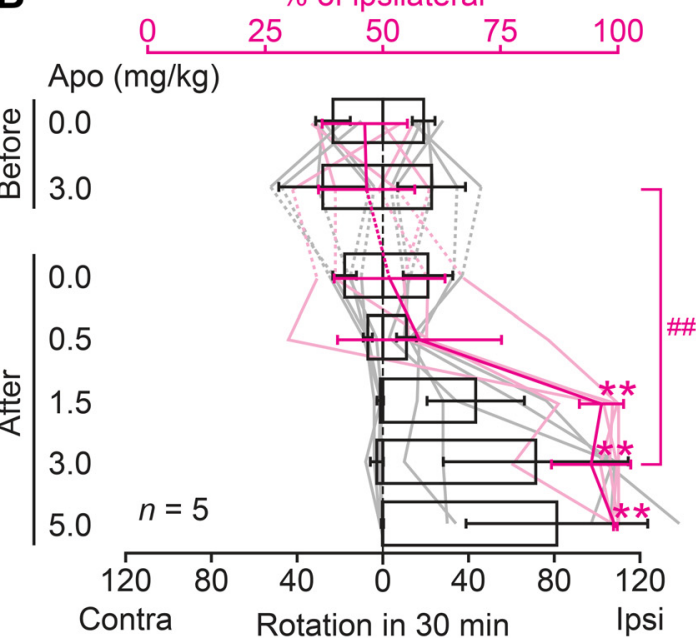

Figure 5. Motor hyperactivity after cortico-STN elimination ( $n$, number of mice tested). $\boldsymbol{A}$, Time course of locomotor activity changes (mean \pm SD) of the control (cyan) and bilaterally eliminated (magenta) mice before and after laser irradiation. The light-colored lines show the data from individual mice. ${ }^{*} p<0.05$ significantly different from the control mice, two-way repeatedmeasures ANOVA with Bonferroni's test. $\boldsymbol{B}$, Numbers of contralateral and ipsilateral rotations to the cortico-STN-eliminated side (mean \pm SD, white bars) in 30 min and the ratios of the ipsilateral rotations to total rotations (mean $\pm S D$, magenta lines) of unilaterally eliminated mice injected with different doses of apomorphine (Apo). The light-colored lines show the numbers of contralateral and ipsilateral rotations (gray) and the ratios of the ipsilateral rotations (light magenta) of individual mice. The ratios of the ipsilateral rotations were compared. ${ }^{* *} p<0.001$ significantly different from $0.0 \mathrm{mg} / \mathrm{kg}$ Apo after cortico-STN elimination, one-way repeated-measures ANOVA with Tukey's test; \#\# $<0.001$ significantly different from one another, two-tailed paired $t$ test

mouse STN using optogenetics has also shown its causal role; brief activation or inhibition of the STN interrupted behaviors or blunted the interruptive effect of surprise, respectively (Fife et al., 2017).

However, STN activity is increased not only in the stop task but also in the go task (Georgopoulos et al., 1983; DeLong et al., 1985; Matsumura et al., 1992; Wichmann et al., 1994). Considering the conduction time of each pathway, the following hypothesis on the function of the cortico-STN hyperdirect pathway is probable (Mink and Thach, 1993; Nambu et al., 2002;
Sano et al., 2013; Chiken et al., 2015). Signals through the hyperdirect pathway first reset the thalamic and cortical activity related to ongoing movements and prepare for the forthcoming signals through the direct pathway, which release an appropriate movement. Finally, signals through the indirect pathway stop all movements. A recent study has also suggested that the GPe can cancel actions through the projection of arkypallidal cells in the GPe back to the striatum (Mallet et al., 2016).

Was the motor hyperactivity observed in this study indeed caused by elimination of the cortico-STN projection and loss 
of signal transmission through the cortico-STN-SNr hyperdirect pathway? The cortico-STN projection is derived from the axon collaterals of small- to medium-sized cortical neurons projecting to other brain regions, especially to the spinal cord (Kita and Kita, 2012). Thus, not only the cortico-STN projection, but also the cortico-spinal projections may have been eliminated, at least in part, after laser irradiation. Furthermore, the STN also projects to the substantia nigra pars compacta $(\mathrm{SNc})$ and striatum, although less abundantly than to the SNr (Parent and Hazrati, 1995). These excitatory inputs from the STN may also be diminished. However, such effects should be negligible because the loss of excitatory inputs from the STN to the spinal cord, SNc, and striatum would cause motor hypoactivity, but not motor hyperactivity as observed in this study.

\section{Pathophysiology of hemiballism}

Previous studies have reported that lesions or inactivation of the STN reduced firing rates, changed the firing patterns of GPi neurons, and induced motor hyperactivity, which is clinically described as hemiballism (Carpenter et al., 1950; Hamada and DeLong, 1992; Vitek et al., 1999; Nambu et al., 2000). The present study showed, however, that the suppression of excitatory cortical inputs to the STN by cortico-STN elimination was sufficient to cause motor hyperactivity without any changes in spontaneous firing rates and patterns of $\mathrm{SNr}$ neurons. Thus, changes in phasic excitatory/inhibitory inputs from the motor cortex to the output stations of the basal ganglia are as important as spontaneous activity changes in the pathophysiology of movement disorders. In line with our present results, it was also reported that limiting glutamatergic transmission in a subpopulation of STN neurons expressing vesicular glutamate transporter 2 caused hyperlocomotion (Schweizer et al., 2014). Thus, the pathophysiological mechanism of hemiballism is considered to be a combination of (1) loss of the reset function by the cortico-STN-SNr/GPi hyperdirect pathway (present study), (2) loss of the stop function by the cortico-striatoGPe-STN-SNr/GPi indirect pathway (Sano et al., 2013), and (3) releasing movements by the firing rate decrease and pauses of SNr/GPi neurons (Carpenter et al., 1950; Hamada and DeLong, 1992; Vitek et al., 1999; Nambu et al., 2000).

\section{Clinical significance}

Our present study also suggests that blockade of the cortico-STN hyperdirect pathway shifts the motor balance toward hyperactivity, and thus may be beneficial for patients with Parkinson's disease. The cortico-STN projection conveys abnormal activity from the motor cortex to the STN in Parkinson's disease (Kita and Kita, 2011; Tachibana et al., 2011). The STN is, indeed, one of the targets of deep brain stimulation (DBS) therapy to ameliorate parkinsonian symptoms (Benabid et al., 2009). A possible therapeutic mechanism of DBS in the STN may be functional blockade of cortico-STN inputs by high-frequency stimulation (Maurice et al., 2003; Moran et al., 2011; Chiken and Nambu, 2014). Such an interpretation is also supported by a study showing that optogenetic stimulation of the cortico-STN projection improved parkinsonian symptoms in mice (Gradinaru et al., 2009). Thus, our study may lead us to better understanding and future advancement of therapeutic intervention in Parkinson's disease.

\section{References}

Alegre M, Lopez-Azcarate J, Obeso I, Wilkinson L, Rodriguez-Oroz MC, Valencia M, Garcia-Garcia D, Guridi J, Artieda J, Jahanshahi M, Obeso JA (2013) The subthalamic nucleus is involved in successful inhibition in the stop-signal task: a local field potential study in Parkinson's disease. Exp Neurol 239:1-12.

Alexander GE, Crutcher MD (1990) Functional architecture of basal ganglia circuits: neural substrates of parallel processing. Trends Neurosci 13:266271.

Benabid AL, Chabardes S, Mitrofanis J, Pollak P (2009) Deep brain stimulation of the subthalamic nucleus for the treatment of Parkinson's disease. Lancet Neurol 8:67-81.

Bevan MD, Wilson CJ (1999) Mechanisms underlying spontaneous oscillation and rhythmic firing in rat subthalamic neurons. J Neurosci 19:76177628.

Carpenter MB, Whittier JR, Mettler FA (1950) Analysis of choreoid hyperkinesia in the Rhesus monkey; surgical and pharmacological analysis of hyperkinesia resulting from lesions in the subthalamic nucleus of Luys. J Comp Neurol 92:293-331.

Chiken S, Nambu A (2014) Disrupting neuronal transmission: mechanism of DBS? Front Syst Neurosci 8:33.

Chiken S, Shashidharan P, Nambu A (2008) Cortically evoked long-lasting inhibition of pallidal neurons in a transgenic mouse model of dystonia. J Neurosci 28:13967-13977.

Chiken S, Sato A, Ohta C, Kurokawa M, Arai S, Maeshima J, SunayamaMorita T, Sasaoka T, Nambu A (2015) Dopamine D1 receptor-mediated transmission maintains information flow through the cortico-striatoentopeduncular direct pathway to release movements. Cereb Cortex 25:4885-4897.

Delfs JM, Ciaramitaro VM, Parry TJ, Chesselet MF (1995) Subthalamic nucleus lesions: widespread effects on changes in gene expression induced by nigrostriatal dopamine depletion in rats. J Neurosci 15:6562-6575.

DeLong MR, Crutcher MD, Georgopoulos AP (1985) Primate globus pallidus and subthalamic nucleus: functional organization. J Neurophysiol 53:530-543.

Do MTH, Bean BP (2003) Subthreshold sodium currents and pacemaking of subthalamic neurons. Neuron 39:109-120.

Dwi Wahyu I, Chiken S, Hasegawa T, Sano H, Nambu A (2021) Abnormal cortico-basal ganglia neurotransmission in a mouse model of L-DOPAinduced dyskinesia. J Neurosci 41:2668-2683.

Eyding D, Macklis JD, Neubacher U, Funke K, Wörgötter F (2003) Selective elimination of corticogeniculate feedback abolishes the electroencephalogram dependence of primary visual cortical receptive fields and reduces their spatial specificity. J Neurosci 23:7021-7033.

Fife KH, Gutierrez-Reed NA, Zell V, Bailly J, Lewis CM, Aron AR, Hnasko TS (2017) Causal role for the subthalamic nucleus in interrupting behavior. Elife 6:e27689.

Georgopoulos AP, DeLong MR, Crutcher MD (1983) Relations between parameters of step-tracking movements and single cell discharge in the globus pallidus and subthalamic nucleus of the behaving monkey. J Neurosci 3:1586-1598.

Gradinaru V, Mogri M, Thompson KR, Henderson JM, Deisseroth K (2009) Optical deconstruction of parkinsonian neural circuitry. Science 324:354-359.

Graybiel AM (2005) The basal ganglia: learning new tricks and loving it. Curr Opin Neurobiol 15:638-644.

Guthrie M, Leblois A, Garenne A, Boraud T (2013) Interaction between cognitive and motor cortico-basal ganglia loops during decision making: a computational study. J Neurophysiol 109:3025-3040.

Hamada I, DeLong MR (1992) Excitotoxic acid lesions of the primate subthalamic nucleus result in transient dyskinesias of the contralateral limbs. J Neurophysiol 68:1850-1858.

Hutchinson WD, Levy R, Dostrovsky JO, Lozano AM, Lang AE (1997) Effects of apomorphine on globus pallidus neurons in parkinsonian patients. Ann Neurol 42:767-775.

Inoue K, Koketsu D, Kato S, Kobayashi K, Nambu A, Takada M (2012) Immunotoxin-mediated tract targeting in the primate brain: selective elimination of the cortico-subthalamic "hyperdirect" pathway. PLoS One 7:e39149.

Isoda M, Hikosaka O (2008) Role for subthalamic nucleus neurons in switching from automatic to controlled eye movement. J Neurosci 28:72097218.

Iwamuro H, Tachibana Y, Ugawa Y, Saito N, Nambu A (2017) Information processing from the motor cortices to the subthalamic nucleus and globus pallidus and their somatotopic organizations revealed electrophysiologically in monkeys. Eur J Neurosci 46:2684-2701. 
Jahfari S, Waldorp L, van den Wildenberg WP, Scholte HS, Ridderinkhof KR, Forstmann BU (2011) Effective connectivity reveals important roles for both the hyperdirect (fronto-subthalamic) and the indirect (frontostriatal-pallidal) fronto-basal ganglia pathways during response inhibition. J Neurosci 31:6891-6899.

Kita H, Kita T (2011) Cortical stimulation evokes abnormal responses in the dopamine-depleted rat basal ganglia. J Neurosci 31:10311-10322.

Kita T, Kita H (2012) The subthalamic nucleus is one of multiple innervation sites for long-range corticofugal axons: a single-axon tracing study in the rat. J Neurosci 32:5990-5999.

Kita H, Nambu A, Kaneda K, Tachibana Y, Takada M (2004) Role of ionotropic glutamatergic and GABAergic inputs on the firing activity of neurons in the external pallidum in awake monkeys. J Neurophysiol 92:3069-3084.

Kravitz AV, Freeze BS, Parker PRL, Kay K, Thwin MT, Deisseroth K, Kreitzer AC (2010) Regulation of parkinsonian motor behaviours by optogenetic control of basal ganglia circuitry. Nature 466:622-626.

Leblois A, Boraud T, Meissner W, Bergman H, Hansel D (2006) Competition between feedback loops underlies normal and pathological dynamics in the basal ganglia. J Neurosci 26:3567-3583.

Legéndy CR, Salcman M (1985) Bursts and recurrences of bursts in the spike trains of spontaneously active striate cortex neurons. J Neurophysiol 53:926-939.

Macklis JD (1993) Transplanted neocortical neurons migrate selectively into regions of neuronal degeneration produced by chromophore-targeted laser photolysis. J Neurosci 13:3848-3863.

Macklis JD, Quattrochi JJ (1991) Restricted diffusion and stability of carbachol-fluorescent nanospheres in-vivo. Neuroreport 2:247-250.

Madison RD, Macklis JD (1993) Noninvasively induced degeneration of neocortical pyramidal neurons in vivo: selective targeting by laser activation of retrogradely transported photolytic chromophore. Exp Neurol 121:153-159.

Madison R, Macklis JD, Thies C (1990) Latex nanosphere delivery system (LNDS): novel nanometer-sized carriers of fluorescent dyes and active agents selectively target neuronal subpopulation via uptake and retrograde transport. Brain Res 522:90-98.

Magavi SS, Leavitt BR, Macklis JD (2000) Induction of neurogenesis in the neocortex of adult mice. Nature 405:951-955.

Mallet N, Schmidt R, Leventhal D, Chen F, Amer N, Boraud T, Berke JD (2016) Arkypallidal cells send a stop signal to striatum. Neuron 89:308316.

Matsumura M, Kojima J, Gardiner TW, Hikosaka O (1992) Visual and oculomotor functions of monkey subthalamic nucleus. J Neurophysiol 67:1615-1632.

Maurice N, Deniau JM, Glowinski J, Thierry AM (1999) Relationships between the prefrontal cortex and the basal ganglia in the rat: physiology of the cortico-nigral circuits. J Neurosci 19:4674-4681.

Maurice N, Thierry AM, Glowinski J, Deniau JM (2003) Spontaneous and evoked activity of substantia nigra pars reticulata neurons during highfrequency stimulation of the subthalamic nucleus. J Neurosci 23:99299936.

Mink JW, Thach WT (1993) Basal ganglia intrinsic circuits and their role in behavior. Curr Opin Neurobiol 3:950-957.

Moran A, Stein E, Tischler H, Belelovsky K, Bar-Gad I (2011) Dynamic stereotypic responses of basal ganglia neurons to subthalamic nucleus highfrequency stimulation in the parkinsonian primate. Front Syst Neurosci 5:21.

Nakanishi H, Kita H, Kitai ST (1987) Electrical membrane properties of rat subthalamic neurons in an in vitro slice preparation. Brain Res 437:3544 .

Nambu A, Takada M, Inase M, Tokuno H (1996) Dual somatotopical representations in the primate subthalamic nucleus: evidence for ordered but reversed body-map transformations from the primary motor cortex and the supplementary motor area. J Neurosci 16:2671-2683.

Nambu A, Tokuno H, Hamada I, Kita H, Imanishi M, Akazawa T, Ikeuchi Y, Hasegawa N (2000) Excitatory cortical inputs to pallidal neurons via the subthalamic nucleus in the monkey. J Neurophysiol 84:289-300.
Nambu A, Tokuno H, Takada M (2002) Functional significance of the cortico-subthalamo-pallidal "hyperdirect" pathway. Neurosci Res 43:111117.

Oldenburg IA, Sabatini BL (2015) Antagonistic but not symmetric regulation of primary motor cortex by basal ganglia direct and indirect pathways. Neuron 87:244

Ozaki M, Sano H, Sato S, Ogura M, Mushiake H, Chiken S, Nakao N, Nambu A (2017) Optogenetic activation of the sensorimotor cortex reveals "local inhibitory and global excitatory" inputs to the basal ganglia. Cereb Cortex 27:5716-5726.

Parent A, Hazrati LN (1995) Functional anatomy of the basal ganglia. II. The place of subthalamic nucleus and external pallidum in basal ganglia circuitry. Brain Res Brain Res Rev 20:128-154

Pasquereau B, Turner RS (2017) A selective role for ventromedial subthalamic nucleus in inhibitory control. eLife 6:e31627.

Paxinos G, Franklin KBJ (2001) The mouse brain in stereotaxic coordinates, Ed 2. San Diego: Academic.

Polyakova Z, Chiken S, Hatanaka N, Nambu A (2020) Cortical control of subthalamic neuronal activity through the hyperdirect and indirect pathways in monkeys. J Neurosci 40:7451-7463.

Redgrave P, Rodriguez M, Smith Y, Rodriguez-Oroz MC, Lehericy S, Bergman H, Agid Y, DeLong MR, Obeso JA (2010) Goal-directed and habitual control in the basal ganglia: implications for Parkinson's disease. Nat Rev Neurosci 11:760-772.

Ryan LJ, Clark KB (1991) The role of the subthalamic nucleus in the response of globus pallidus neurons to stimulation of the prelimbic and agranular frontal cortices in rats. Exp Brain Res 86:641-651.

Sano H, Yasoshima Y, Matsushita N, Kaneko T, Kohno K, Pastan I, Kobayashi K (2003) Conditional ablation of striatal neuronal types containing dopamine D2 receptor disturbs coordination of basal ganglia function. J Neurosci 23:9078-9088.

Sano H, Chiken S, Hikida T, Kobayashi K, Nambu A (2013) Signals through the striatopallidal indirect pathway stop movements by phasic excitation in the substantia nigra. J Neurosci 33:7583-7594.

Scharff C, Kirn JR, Grossman M, Macklis JD, Nottebohm F (2000) Targeted neuronal death affects neuronal replacement and vocal behavior in adult songbirds. Neuron 25:481-492.

Schmidt R, Leventhal DK, Mallet N, Chen F, Berke JD (2013) Canceling actions involves a race between basal ganglia pathways. Nat Neurosci 16:1118-1124.

Schweizer N, Pupe S, Arvidsson E, Nordenankar K, Smith-Anttila CJ, Mahmoudi S, Andrén A, Dumas S, Rajagopalan A, Lévesque D, Leão RN, Wallén-Mackenzie Å (2014) Limiting glutamate transmission in a Vglut2-expressing subpopulation of the subthalamic nucleus is sufficient to cause hyperlocomotion. Proc Natl Acad Sci U S A 111:7837-7842.

Sheen VL, Macklis JD (1994) Apoptotic mechanisms in targeted neuronal cell death by chromophore-activated photolysis. Exp Neurol 130:67-81.

Tachibana Y, Kita H, Chiken S, Takada M, Nambu A (2008) Motor cortical control of internal pallidal activity through glutamatergic and GABAergic inputs in awake monkeys. Eur J Neurosci 27:238-253.

Tachibana Y, Iwamuro H, Kita H, Takada M, Nambu A (2011) Subthalamopallidal interactions underlying parkinsonian neuronal oscillations in the primate basal ganglia. Eur J Neurosci 34:1470-1484.

Vitek JL, Chockkan V, Zhang JY, Kaneoke Y, Evatt M, DeLong MR, Triche S, Mewes K, Hashimoto T, Bakay RA (1999) Neuronal activity in the basal ganglia in patients with generalized dystonia and hemiballismus. Ann Neurol 46:22-35.

Waszczak BL, Martin LP, Finlay HE, Zahr N, Stellar JR (2002) Effects of individual and concurrent stimulation of striatal D1 and D2 dopamine receptors on electrophysiological and behavioral output from rat basal ganglia. J Pharmacol Exp Ther 300:850-861

Wichmann T, Bergman H, DeLong MR (1994) The primate subthalamic nucleus. I. Functional properties in intact animals. J Neurophysiol 72:494506.

Wichmann T, DeLong MR, Guridi J, Obeso JA (2011) Milestones in research on the pathophysiology of Parkinson's disease. Mov Disord 26:10321041. 